\title{
Percepción de actores locales sobre implicancias de la focalización en el Valle del Mantaro
}

Perception of local actors on implications of targeting in the Mantaro Valley

Aparicio Chanca Flores ${ }^{1}$

RECIBIDO: 9 DE febrero DE 2020

ACEPTADO: 5 DE JUNIO DE 2020

\section{RESUMEN}

El Sistema de Focalización de Hogares (SISFOH) es un proceso de determinación de la clasificación socioeconómica (CSE) que certifica a las personas y grupos poblacionales vulnerables, sumidos en la pobreza o extrema pobreza, ante los programas sociales y subsidios del Estado. El objetivo de esta investigación es mostrar la percepción que tienen los actores locales sobre las implicancias de la focalización en el Valle del Mantaro. Es de tipo básico y de nivel descriptivo, tiene como unidades de análisis a pobladores no beneficiarios de ocho distritos que forman parte de las

\footnotetext{
1 Doctor en Ciencias ambientales y desarrollo sostenible, Magister en Desarrollo Rural, Licenciado en Antropología, Docente, Universidad Nacional del Centro del Perú, Huancayo, Perú; achanca@uncp.edu.pe, ORCID: https://orcid.org/0000-0002-5664-5444

Google Scholar: https://scholar.google.com/scholar?hl=es\&as_sdt=0\%2C5\&q=aparicio+chanca\&oq=apari
} 


\title{
64
}

cuatro provincias del Valle del Mantaro, escenario en donde se aplicó 384 cuestionarios basados en una escala de Likert, a personas mayores de 15 años. El análisis de datos se realizó en el software SPSS 25.0, obteniéndose como resultado que los actores locales, en su mayoría, tienen una percepción negativa sobre el SISFOH, porque observan ciertas limitaciones de inclusión y exclusión en el registro y certificación.

Palabras clave: percepción, actores locales, programa social, focalización

\begin{abstract}
The Household Targeting System (in Spanish, SISFOH) is a process for determining the Socioeconomic Classification (SEC), which certifies vulnerable people and population groups, inmersed in poverty or extreme poverty, in the face of state social programs and subsidies. The research's objective is to show the perception that local actors have about the implications of targeting in Mantaro Valley. It is a basic type and descriptive lever research, it has as analysis units non-beneficiary residents of eight districts that are part of the four provinces of Mantaro Valley. Were surveyed 384 persons over 15 years-old, with an Likert scale. Data analysis was performed in software SPSS 25.0, obtaining as a result that the vast majority have a negative perception of the SISFOH, because they observe some limitations of inclusion and exclusion in the registry and certification.
\end{abstract}

Keywords: perception, local actors, social program, targeting

\section{Introducción}

Según Brodersohn (1999) la focalización surge en América Latina en la década del 80, la cual inicialmente fue cuestionada por su contenido político e ideológico (p. 1). Por su parte, Sojo (2007) señala que en 1970 ya se hablaba de focalización y estaba relacionada con los planteamientos de redistribución con crecimiento (p. 113). La focalización es un instrumento, no un objetivo de política (Rosero, 2004, p. 58), su finalidad es alcanzar eficiencia, eficacia y equidad en la ejecución de programas sociales y subsidios del Estado, que permitan garantizar el bienestar del individuo 
(Ravallion y Lokshin, 1999, p. 6). Por ello, como señala Raczynski (1995), este término se viene utilizando en diversas situaciones que impliquen priorizar, delimitar y decidir o definir un blanco, una acción o meta (p. 217)

Por otra parte, Cecchini y Madariaga (2011 , p. 13) y Regalia y Stampini (2017, p. VIII) realizan estudios sobre los programas de transferencia condicionada en un total de 18 países de América Latina y el Caribe sobre la entrega de recursos monetarios y no monetarios a familias en situación de pobreza y pobreza extrema. Oyarzún y Soto (2018) reflexionan sobre la política pública en la educación escolar chilena a partir del subsidio focalizado en grupos de nivel socioeconómico bajo, que no muestra mejoras académicas, pero sí incremento de alumnos (p. 200). Mientras que Oliveri (2016) investiga acerca de la relación que existe entre las pensiones y la pobreza en la vejez en 17 países de América Latina (p. 124). Hay que tener en cuenta, sin embargo, lo que señalan Gonzales (2017, p. 275) y Chanca (2020, p. 80): que la focalización muchas veces conlleva a la estigmatización y errores de inclusión o exclusión de la población objetiva.

Como manifiesta Lavalleja y Tenenbaum (2020), se utilizan diversos criterios y mecanismos de selección de individuos $u$ hogares (p. 9). Asimismo, como han planteado Cerón y Raccanello (2018), en México se utiliza el índice de desarrollo social como herramienta de focalización (pp. 64-65), y el sistema multidimensional para medir la pobreza (Aguilar, Caamal y Ortíz, 2018, p. 106). En Colombia, se utiliza la estratificación socioeconómica como instrumento de focalización para identificar hogares carentes de servicios públicos domiciliarios como refieren Alzate (2006, p. 11) y Rosero (2004, p. 54). Pero cabe resaltar lo que revela Chudnovsky (2019), que este tipo de enfoques trae problemas de exclusión de ciudadanos que realmente necesitan los recursos y la inclusión de otros que no forman parte de este grupo poblacional (p. 8), considerando de esta forma al focalizado como "un receptor pasivo, más que la de un agente activo"(Sen, 2003, p. 555).

Para el caso peruano el Sistema de Focalización de Hogares (SISFOH), es una instancia que proporciona información socioeconómica básica obtenida mediante un conjunto de criterios, reglas o procedimientos, a las diversos programas sociales y subsidios del Estado (MIDIS, 2012; MIDIS, 2015), los mismos que se vienen ejecutando en todo el país. Estos programas y subsidios hacen uso de los datos proporcionados 


\section{6}

por el SISFOH para identificar y priorizar a sus beneficiarios, con la finalidad de garantizar la entrega de los recursos públicos con equidad y eficiencia al grupo poblacional priorizado, que se halla en condiciones de pobreza o pobreza extrema. En diciembre de 2004 se aprueba la Política Nacional de Focalización bajo rectoría de la Presidencia del Consejo de Ministros - PCM mediante D.S. № 399-2004-EF, que da lugar a la creación del SISFOH con R.M. N 399-2004-PCM. Luego, en octubre de 2005, se transfiere a la Dirección General de Asuntos Económicos y Sociales del Ministerio de Economía y Finanzas MEF con R.M. N³72-2005-PCM, a partir de lo cual se inició su implementación progresiva. Tiene como como finalidad proporcionar información socioeconómica actualizada a los programas sociales para que identifiquen y seleccionen a sus beneficiarios (MIDIS, 2017).

Posteriormente, con la creación del Ministerio de Inclusión Social (MIDIS) con Ley N²9792 en 2011, se aprueba, mediante Decreto de Urgencia No 001-2012, que el SISFOH sea transferido a esta instancia y a continuación, con la "Ley de Presupuesto del Sector Público para el Año Fiscal 2013" № 29951, se dispone que la Dirección de Operaciones (DO), a cargo del SISFOH, esté bajo el ámbito de la Dirección General de Focalización e Información Social (DGFIS) del Ministerio de Desarrollo e Inclusión Social, de manera permanente. Posteriormente, en 2016, con la Ley $N^{\circ} 30435$, se aprueba la creación del Sistema Nacional de Focalización (SINAFO), que establece normatividades, guías y procedimientos, orientados a regular la focalización de hogares y actualización del padrón general de hogares, para que todo programa social o subsidio del Estado utilice la evaluación del SISFOH para elegir sus usuarios. Todo esto, en el marco de la directiva Nº06-2017-MIDIS.

En el Perú, existen dos mecanismos o tipos de focalización. El primer mecanismo está referido a la focalización de hogares o por individuos que se desarrolla en función a las características socioeconómicas, demográficas y condiciones de vida; mientras que el segundo mecanismo es la focalización geográfica, encargada de identificar áreas geográficas que concentran población en situación de pobreza, elabora mapas de pobreza del país, según regiones, provincias y distritos. Además, existen dos criterios de focalización para seleccionar y afiliar a la población objetiva: la socioeconómica y la categórica. La primera está referida a la condición de pobreza de la población o del individuo que se halla registrada en el padrón general de 
hogares, mientras que la segunda está referida a otras características específicas que están relacionadas a los objetivos de intervención de los programas sociales y subsidios. Así, el SISFOH brinda los criterios socioeconómicos, mientras que los programas sociales se encargan de los criterios categóricos según los perfiles o protocolos establecidos.

El objetivo de este documento es mostrar la percepción que tienen los actores locales no beneficiarios sobre las implicancias de la focalización de beneficiarios de los programas sociales que tuvo lugar en ocho distritos de cuatro provincias del Valle del Mantaro de la región Junín. Sobre el tema propuesto, existen bibliografías en informes institucionales, artículos científicos, tesis de grado y otros desde la perspectiva del beneficiario o del ejecutor, pero hay limitada bibliografía referido a la percepción de los no beneficiarios del programa, hecho que compromete a realizar la investigación.

Según MIDIS (2012), el primer paso para garantizar que el aporte de los recursos del Estado llegue a todas las personas que lo necesiten y solo a ellas es "identificarlas en todo el territorio nacional, y determinar qué programa social o qué combinación de intervenciones de política social se requiere para empezar su proceso de inclusión social, focalizarlas y afiliarlas". Por lo tanto, según esta metodología que el MIDIS establece, la primera tarea es la de focalización de hogares y la segunda el proceso de afiliación. Es un conjunto de reglas e instrumentos que permiten identificar a personas o grupos poblacionales en situación de pobreza, vulnerabilidad o exclusión, como potenciales beneficiarios de los programas sociales y subsidios del Estado Peruano.

\section{Método}

El presente estudio corresponde a una investigación básica de nivel descriptivo, que tuvo lugar en ocho distritos de las cuatro provincias que forman parte del Valle del Mantaro, entre ellas: Acolla y Jauja en la provincia de Jauja; San Jerónimo y Concepción en la provincia de Concepción; Ahuac y Chupaca en la provincia Chupaca; y El Tambo y Chilca en la provincia de Huancayo. Esta selección obedece a que los distritos mencionados poseen el mayor número de beneficiarios de los programas sociales. 


\section{8}

La población considerada para el estudio fue de varones y mujeres mayores de 15 años, de ocho distritos de las cuatro provincias del valle del Mantaro. Según la clasificación de Ramírez y Fernández (2013), esta selección etaria corresponde a los grupos poblacionales jóvenes de 16 a 24 años, adultos de 25 a 59 años y adultos mayores de 60 años a más (p. 72), principales actores locales, cuya participación es consentida y tomada en cuenta en las diversas acciones que realizan a nivel familiar y comunal en cada distrito.

La muestra estuvo compuesta por 384 actores locales mayores de 15 años, quienes participaron voluntariamente en el estudio durante la visita realizada a sus domicilios. Los actores locales cuentan con las siguientes características sociodemográficas:

- Sexo: Varones: $43,8 \%$ - Mujeres: $56,2 \%$.

- Grupos de edades: Jóvenes: 34,4\% - Adultos: 44,2\% - Adultos mayores: 21,4\%.

- Nivel educativo: Analfabetos: 7,8\% - Primaria: 17,7\% - Secundaria: 32,8\% Superior técnico: 14,1\% - Superior universitaria: $27,6 \%$.

Se considera un nivel de confianza de $95 \%$ y un margen de error de $5 \%$, establecido según el muestreo probabilístico bajo los principios de equiprobabilidad, según el cual cada actor local mayor de 15 años de edad tuvo la misma oportunidad de ser encuestado.

Para recoger datos de campo se utilizó un cuestionario cuya fiabilidad se determinó a través de a de Cronbach (Hernández, Fernández y Baptista, 2014, p. 208), obteniéndose un coeficiente de 0,87 , lo que indica una buena fiabilidad del instrumento. La técnica utilizada fue una encuesta elaborada según la escala de Likert con 10 reactivos, todos con cinco opciones ("Muy mala", "Mala", "Regular", "Buena" y "Muy buena"). La encuesta se aplicó a una muestra de 384 personas no beneficiarias de los diversos programas sociales que se viene ejecutando en el Valle del Mantaro. Además, estos datos fueron complementados con entrevistas realizadas a los principales actores locales de cada ámbito distrital priorizado.

Para la aplicación del cuestionario, se contó con el apoyo de dos estudiantes de Antropología, quienes antes de salir a campo fueron capacitados en el uso y manejo del instrumento y luego de hacer un recorrido por cada uno de los distritos priorizados, realizando visitas casa por casa, así como a las oficinas de las principales autoridades y funcionarios distritales a quienes, además de ser encuestados, se entrevistó. 
El trabajo de campo permitió para generar una base de datos en el programa estadístico SPSS 25.0. Dicha base de datos permitió medir la predominancia de la percepción de los actores locales no beneficiarios sobre las implicancias de la focalización.

\section{Resultados}

Percepción de los actores locales sobre el proceso de focalización en ocho distritos de cuatro provincias del Valle del Mantaro.

\section{Tabla 1}

Percepción sobre el sistema de focalización de hogares en el Valle del Mantaro

\begin{tabular}{lcccc}
\hline \hline & Frecuencia & Porcentaje & $\begin{array}{c}\text { Porcentaje } \\
\text { válido }\end{array}$ & $\begin{array}{c}\text { Porcentaje } \\
\text { acumulado }\end{array}$ \\
\hline Muy mala & 56 & 14,6 & 14,6 & 14,6 \\
Mala & 96 & 25,0 & 25,0 & 39,6 \\
Regular & 150 & 39,1 & 39,1 & 78,6 \\
Buena & 78 & 20,3 & 20,3 & 99,0 \\
Muy buena & 4 & 1,0 & 1,0 & 100,0 \\
Total & 384 & 100,0 & 100,0 & \\
\hline \hline
\end{tabular}

De un total de 384 personas encuestadas en los ocho distritos de las cuatro provincias del Valle del Mantaro sobre el SISFOH, un 39,1\% considera que el proceso es regular, seguido por un $25 \%$ que lo considera malo y un $14,6 \%$ muy malo, mientras que un $20,3 \%$ lo considera bueno y, finalmente, un $1 \%$ lo considera muy bueno. Este resultado nos muestra que la mayoría de los pobladores perciben negativamente este proceso, por ser muy engorroso y burocrático, aun tratándose de personas que se hallan en pobreza o extrema pobreza, a diferencia de los que consideran el proceso como bueno y muy bueno, quienes refieren que este proceso es el más adecuado para seleccionar a los más necesitados. 
70

\section{Tabla 2}

Percepción sobre presentación y registro de solicitud al Sistema de Focalización de Hogares-SISFOH

\begin{tabular}{lcccc}
\hline \hline & Frecuencia & Porcentaje & $\begin{array}{c}\text { Porcentaje } \\
\text { válido }\end{array}$ & $\begin{array}{c}\text { Porcentaje } \\
\text { acumulado }\end{array}$ \\
\hline Muy mala & 34 & 8,9 & 8,9 & 8,9 \\
Mala & 96 & 25,0 & 25,0 & 33,9 \\
Regular & 159 & 41.4 & 41.4 & 75,3 \\
Buena & 80 & 20,8 & 20,8 & 96,1 \\
Muy buena & 15 & 3,9 & 3,9 & 100,0 \\
Total & 384 & 100,0 & 100,0 & \\
\hline \hline
\end{tabular}

En la tabla 2. se observa que el 41,4\% de los pobladores del Valle del Mantaro perciben que la presentación y registro de solicitud en el formato $\$ 100$ de clasificación socioeconómica CSE a la unidad local de empadronamiento - ULE en la Municipalidad distrital donde vive es regular, siendo la percepción predominante, seguida por un $25 \%$ que la considera mala, porque según los entrevistados, para este proceso se requiere una serie de requisitos que el solicitante debe cumplir y algunos de ellos tienen un costo y no pueden obtenerlo por falta de dinero.

\section{Tabla 3}

Percepción sobre empadronamiento, recojo y registro de datos en Ficha Socioeconómica Única FSU

\begin{tabular}{lcccc}
\hline \hline & Frecuencia & Porcentaje & $\begin{array}{c}\text { Porcentaje } \\
\text { válido }\end{array}$ & $\begin{array}{c}\text { Porcentaje } \\
\text { acumulado }\end{array}$ \\
\hline Muy mala & 18 & 4,7 & 4,7 & 4,7 \\
Mala & 98 & 25,5 & 25,5 & 30,2 \\
Regular & 152 & 39,6 & 39,6 & 69,8 \\
Buena & 98 & 25,5 & 25,5 & 95,8 \\
Muy buena & 18 & 4,7 & 4,7 & 100,0 \\
Total & 384 & 100,0 & 100,0 & \\
\hline \hline
\end{tabular}


La tabla 3, muestra que casi el $40 \%$ de los encuestados perciben que el proceso de empadronamiento de la ULE que funciona en los municipios (la cual es la encargada de recoger los datos de los futuros focalizados in situ, en el domicilio del solicitante, donde llena la ficha para luego registrarla electrónicamente en la Ficha Socioeconómica Única-FSU, la que se envía al MIDIS para que aplique el Índice de Focalización de Hogares) es considerada regular, siendo esta la percepción predominante. También se observa una percepción equilibrada de percepción buena y mala con un porcentaje de $25,5 \%$, así como la percepción de muy buena y muy mala con un valor de $4,7 \%$ respectivamente. Este resultado que no concuerda con lo obtenido mediante las entrevistas, en las cuales la mayoría señala que estos actos responden a intereses o favores políticos, en los cuales son priorizados los familiares, amistades o simpatizantes del grupo político que se halla en el poder del gobierno local, dejando de lado a los que verdaderamente requieren.

\section{Tabla 4}

Percepción sobre determinación y registro de la clasificación socioeconómica CSE

\begin{tabular}{lcccc}
\hline \hline & Frecuencia & Porcentaje & $\begin{array}{c}\text { Porcentaje } \\
\text { válido }\end{array}$ & $\begin{array}{c}\text { Porcentaje } \\
\text { acumulado }\end{array}$ \\
\hline Muy mala & 14 & 3,6 & 3,6 & 3,6 \\
Mala & 100 & 26,0 & 26,0 & 29,7 \\
Regular & 174 & 45,3 & 45,3 & 75,0 \\
Buena & 86 & 22,4 & 22,4 & 97,4 \\
Muy buena & 10 & 2,6 & 2,6 & 100,0 \\
Total & 384 & 100,0 & 100,0 & \\
\hline \hline
\end{tabular}

En la tabla 4. se observa que más del $45 \%$ de los pobladores encuestados en el Valle del Mantaro perciben que el proceso de determinación y registro de la CSE es regular, siendo la percepción predominante. Además, el 26\% tiene una percepción negativa sobre este proceso, calificándola de mala, a pesar de que el MIDIS es la encargada de evaluar la coherencia de la información recogida en el formato de solicitud S100 y la ficha socioeconómica única FSU. Estos resultados reportados en primera instancia, permiten a la autoridad local, suscribir la declaración jurada en 
formato S100, la cual reportan mediante el mecanismo electrónico al MIDIS, instancia que evalúa y determina el registro del resultado de la CSE de la solicitud en el padrón general de hogares PGH.

Los que perciben de forma negativa este proceso consideran que el MIDIS solo ratifica lo priorizado por los trabajadores de la ULE, quienes cumplen el encargo del alcalde por ser personal de confianza, razón por la cual se observa como beneficiarios de estos programas a personas que cuentan con negocios, ganados, terrenos agrícolas o buenas casas, mientras que otras personas que verdaderamente requieren su inclusión en el programa no están. En otros casos los responsables de la Municipalidad señalan que, a pesar de que los solicitantes cumplen con todos los requisitos, el MIDIS no los prioriza en el padrón general de hogares PGH.

\section{Tabla 5}

Percepción sobre certificación y acceso al resultado de la clasificación socioeconómica CSE

\begin{tabular}{lcccc}
\hline \hline & Frecuencia & Porcentaje & $\begin{array}{c}\text { Porcentaje } \\
\text { válido }\end{array}$ & $\begin{array}{c}\text { Porcentaje } \\
\text { acumulado }\end{array}$ \\
\hline Muy mala & 41 & 10,7 & 10,7 & 10,7 \\
Mala & 88 & 22,9 & 22,9 & 33.6 \\
Regular & 150 & 39.0 & 39.0 & 72,6 \\
Buena & 89 & 23.2 & 23.2 & 95,8 \\
Muy buena & 16 & 4,2 & 4,2 & 100,0 \\
Total & 384 & 100,0 & 100,0 & \\
\hline \hline
\end{tabular}

En la tabla 5 se observa que el 39\% de los pobladores encuestados, perciben que la Dirección de Operaciones de Focalización (DOF) del MIDIS (encargada de certificar ante las intervenciones públicas que lo requieran y el acceso a los resultados de la CSE) es regular, siendo la percepción predominante. Además, se observa un 23,2\% la considera como buena, seguido muy de cerca por un 22,9\% que lo considera mala. Quienes perciben como buena a esta Dirección, refieren que existen diversos espacios para que los solicitantes puedan enterarse del resultado obtenido, desde el 
uso de la página web, la misma Municipalidad o en los módulos de intervención pública focalizada. Mientras que los que la perciben como mala, manifiestan que muchos solicitantes que verdaderamente requieren de estos beneficios no obtienen esta certificación por no cumplir con presentar algún requisito exigido por el SISFOH.

\section{Discusión}

Según la literatura revisada para esta investigación, la focalización nace en la década del 80 en América Latina (Brodersohn, 1999, p. 1). Su finalidad es alcanzar eficiencia, eficacia y equidad en el uso de los recursos económicos asignados a programas sociales y subsidios del Estado, para garantizar el bienestar del individuo (Ravallion y Lokshin, 1999, p. 6). Está dirigido a los sectores más vulnerables del país, para que permita salir del embrollo de la marginación y pobreza en que se halla sumida una parte de la población. Pero este objetivo aún tiene limitaciones en el proceso de clasificación socioeconómica (CSE), como la inclusión de beneficiarios que no corresponden al perfil, situación denominada como "filtración", además de provocar la exclusión de individuos que sí requieren ser incluidos (Gonzales, 2017, p. 275), situación denominada "subcobertura" y que permite el clientelismo político.

Los datos encontrados en los ocho distritos del Valle del Mantaro nos muestran que para casi el $40 \%$ de los encuestados, el SISFOH es considerado como regular, mientras que el $25 \%$ lo considera malo, seguido por casi el $15 \%$ que lo considera muy malo, datos que muestran una tendencia negativa de parte de los actores locales, lo que coincide con los resultados obtenidos en Chile por Oyarzún y Soto (2018, p. 198). Cabe resaltar que los entrevistados consideran que la focalización está directamente relacionada con los programas sociales los cuales son, según ellos, una "gastadera de plata", porque los beneficiarios se están acostumbrando al "facilismo y al ocio" al recibir diversos apoyos del Estado, apoyos que, por otra parte, no cumplen con los objetivos para los cuales fueron creados (Chanca, 2020, p. 80), porque la población beneficiaria continúa sumida en la pobreza y extrema pobreza y no se observa mejoría en las condiciones de vida de estas familias.

Por otra parte, un representativo $20 \%$ de los encuestados considera que la focalización es buena porque refieren que es un gran apoyo a las personas y familias vulnerables que se hallan en estado de pobreza y extrema pobreza. 


\section{4}

Sobre las etapas que considera el SISFOH sobre el proceso de determinación de la Clasificación Socioeconómica (CSE), se observa que un porcentaje cercano al 50\% de los encuestados coincide en catalogarla de regular, una categoría intermedia que fue considerada en el cuestionario, resultado que denota la ambivalencia o indiferencia (Hernández, Espejo, Gonzales y Gómez, 2001, p. 137) o como señalan Rojas y Fernández (2000), la duda o indecisión de parte de los encuestados (p. 171). Este hecho permitió dejar estos resultados y utilizar la segunda categoría según el porcentaje obtenido para mostrar las implicancias de la focalización.

El $25 \%$ de los encuestados considera que la presentación y registro de la solicitud es mala, seguido por casi un $21 \%$ que la considera buena. Según los primeros, para esta etapa se requiere una serie de requisitos documentados que el solicitante debe cumplir, proceso similar al de México, Colombia, Bolivia, Uruguay Ecuador y otros países de América Latina y el Caribe como señalan Cecchine y Madariaga (201 1, p. 29), Regalia y Stampini (2017, p. 26) y Lavalleja y Tenenbaum (2020, p. 9). Algunos de estos requisitos requieren ser tramitados en diversas instancias, lo que implica realizar pagos que muchas veces no están al alcance de estas familias.

El empadronamiento que considera el recojo de datos en el hogar y el registro de la Ficha Socioeconómica, reporta datos de igualdad entre los que responden positiva y negativamente un $30,2 \%$, cada uno en forma acumulada, resultado que no coincide con lo obtenido en las entrevistas, donde la mayoría señala que estos actos responden a intereses o favores políticos, en los cuales se prioriza a familiares, amistades o simpatizantes del grupo político que se halla en el poder local. Esta actitud conlleva a dejar desapercibidos a quienes verdaderamente requieren del programa, como manifiestan Chudnovsky (2019,p . 8) y Rosero (2004, p. 65). Quienes opinan a favor, por otra parte, manifiestan que son procesos que garantizan una verdadera selección de los mas necesitados.

Sobre el proceso de determinación y registro de la Clasificación Socioeconómica CSE, el $26 \%$ de los encuestados tiene una percepción negativa, calificándola de mala, seguido por un poco más del $22 \%$ que muestra actitud positiva, considerándola buena. El primer grupo consideran que el MIDIS es una instancia que solo ratifica lo priorizado por la ULE, y estos a la vez cumplen órdenes de las autoridades municipales, por ser personal de confianza. Señalan además que en cada gestión 
del gobierno local aparecen nuevos beneficiarios que son familiares o amistades de los alcaldes, regidores o funcionarios y que se convierten en beneficiario sin reunir los requisitos. Los responsables de las ULEs señalan que ellos cumplen con reportar al MIDIS los documentos de todos los solicitantes, según requisitos exigidos, esperando su priorización en el padrón general de hogares PGH.

Por último, en lo referido a la certificación y acceso al resultado de la CSE, también se observa un resultado casi equilibrado de un $23.2 \%$ que la considera buena, seguido muy de cerca por un $22,9 \%$ que la considera mala. El primer grupo refiere que existen diversos espacios para que los solicitantes puedan enterarse del resultado obtenido, desde la página web, la misma Municipalidad o en los módulos de intervención pública focalizada. Por su parte, el segundo grupo manifiesta que muchos solicitantes que requieren de estos beneficios no obtienen la certificación por no presentar algún requisito exigido por el SISFOH, situación que, según ellos, debe ser apoyada por el alcalde.

Los resultados obtenidos en las diversas etapas que considera el proceso de Clasificación Socioeconómica CSE, responden a una política internacional que es planteada por el Banco Mundial. Como señalan Lavalleja y Tenenbaum (2020), de 29 programas de transferencia condicionada que se ejecutan en veinte países, el 71\%, utiliza como mecanismo la focalización (p. 10).

Para hablar de focalización es necesario entender que la pobreza aún es considerada en la agenda mundial de los organismos internacionales, gobiernos nacionales, regionales y locales así como en académicos e investigadores quienes siguen preocupados en analizar, interpretar y proponer alternativas de solución frente a los criterios de distanciamiento social entre los denominados países ricos y pobres, desarrollados y subdesarrollados, centrales y periféricos, nominaciones cotidianas que se oyen en diversas instancias para mostrar las diferencias de la calidad de vida en que se hallan los habitantes de cada país.

\section{Conclusiones}

En todas las tablas que se muestra, se observa la prevalencia de una percepción regular, con un porcentaje cercano al 50\%, una categoría intermedia que genera ambivalencia o indecisión de los encuestados, seguido por la mayoría que lo 


\section{6}

considera mala, resultado que permite señalar que aún existen limitaciones o falencias de inclusión y exclusión en el proceso de clasificación socioeconómica del SISFOH.

Es menester manifestar también, que la mayoría de los entrevistados refiere que este proceso es muy engorroso y burocrático, tratándose aún de un sector vulnerable que requiere apoyo por presentar limitaciones, no solo en el aspecto económico sino además en procesos de gestión y documentación y así lograr su registro y certificación, por lo que proponen disminuir el clientelismo político, que está presente en el gobierno local.

Con las respuestas vertidas en las entrevistas realizadas se puede afirmar que la focalización de beneficiarios, si bien presenta insatisfacciones, durante los últimos años el sistema de trabajo de las instituciones responsables ha ido mejorando, como refieren la mayoría de los actores locales de los ocho distritos, porque hoy se puede visualizar que gente de escasos recursos económicos está siendo atendida y beneficiada con el programa, repercutiendo en una mejora en la calidad de vida.

\section{Referencias}

Aguilar-Estrada, A., Caamal-Cahuich, I. y Ortiz-Rosales, M. (2018). Pobreza multidimensional en Chiapas: generalizada pero heterogénea. Estudios Sociales y Humanísticos, XVI, 105-117.

Alzate, C. (2006). La estratificación socioeconómica para el cobro de los servicios públicos domiciliarios en Colombia ¿̇Solidaridad o focalización. In CEPAL - Serie Estudios y perpectivas (Vol. 14).

Brodersohn, V. (1999). Focalización de programas de superación de la pobreza. Derecho a Tener Derecho: Infancia, Derecho y Política Social En América Latina., 1-25.

Cecchini, S., Madariaga, A. (2011). Programas de transferencias condicionadas. Balance de la experiencia reciente en América Latina y El Caribe. Santiago de Chile: CEPAL, Naciones Unidas.

Cerón Vargas, J. A., y Raccanello, K. (2018). Índice de desarrollo social de la Ciudad de México como herramienta de focalización de la política social. Retos de La Dirección, 12(2), 64-86. 
Chanca Flores, A. (2020). Percepción cultural de los pobladores sobre el Programa Social Juntos en el Valle del Mantaro. Socialium, 4(1), 68-83. https://doi.org/10.31876/sl.v4i1.49

Chudnovsky, M. (2019). Mas allá de la Focalización de la política pública: Incidencia de factores extra organizacionales en la falta de cobertura universal por hijo en Argentina. Revista Estado, Gobierno y Gestión Pública, 33, 5-37.

Gonzales, R. (2017). Disyuntiva entre universalidad y focalización de las políticas sociales. Revista MERCOSUR de Políticas Sociales, 1, 269. https://doi.org/10.28917/ism.2017-v1-269

Hernández, A., Espejo, B., Gonzales, V. y Gömez, J. (2001). Escalas de respuesta tipo Likert: ¿̇s relevante la alternativa "indiferente"? Metodología de Encuestas, $3(2), 135-150$.

Hernández, R., Fernández, C. y Baptista, M. (2014). Metodología de la investigación. Sexta Edición. México: McGraw-Hill, Interamericana Editores.

Lavalleja, M. y Tenenbaum, V. (2020). Mecanismos de focalización de los programas de transferencias monetarias en América Latina: El caso Uruguay. CEPAL Naciones Unidas.

Ministerio de Desarrollo e Inclusión Social. (2017). Resolución Ministerial N.o 278-2017MIDIS. Manual de operaciones del Programa Juntos. Recuperado de http://www2.juntos.gob.pe/docs/Portal_transparencia/procesos/ Manual_Operaciones/R.M_278-2017-MIDIS_Manual_operaciones.pdf

Ministerio de Desarrollo e Inclusión Social. (2013). Estrategia Nacional de Desarrollo e Inclusión Social "Incluir para crecer". Recuperado de http:// www.midis.gob.pe/dmdocuments/estrategia_incluircrecer.pdf

Ministerio de Desarrollo e Inclusión Social. (2012). Estrategia Nacional de Desarrollo e Inclusión Social. 92. www.midis.gob.pe

Oliveri, M. L. (2016). Pensiones sociales y pobreza en América Latina TT - Social Pensions and Poverty in Latin America. InterAmerican Development Bank, Washington D.C., 43(78), 121-157. https://doi.org/10.21678/0252-186500430078_5

Oyarzún, C., y Soto, R. (2018). Subvención Escolar Preferencial en Chile: ¿descentralización focalizada para el mejoramiento escolar? International 
Journal of Sociology of Education, $7(2)$, 181-208. https://doi.org/10.17583/rise.2018.3321

Raczynski, D. (1995). Focalización de programas sociales: Lecciones de la experiencia chilena. Reasearch Gate, June, 217-256.

Ramírez, J. y Fernández, F. (2013). Paisaje e identidad en El Arbolito, Pachuca, Hidalgo. Jóvenes y adultos en apego a un barrio ex minero. Investigaciones Geográficas, Boletín del Instituto de Geografía, UNAM, ISSN 0188-4611, 80, pp. 71-85.

Ravallion, M. y Lokshin, M. (1999). Subjective Economic Welfare. World Bank Policy Research Working Paper, 2106, 1-39.

Regalia, F., y Stampini, M. (2017). Así funcionan las transferencias condicionadas: Buenas prácticas a 20 años de implementación. En Así funcionan las transferencias condicionadas: Buenas prácticas a 20 años de implementación. https://doi.org/10.18235/0000631

Rojas, A.; Fernández, J. (2000). Análisis de las alternativas de respuesta intermedias mediante el modelo de escalas de clasificación. Metodología de Encuestas, 2(2), 171-184.

Rosero, L. M. (2004). Estratificación socioeconómica como instrumento de focalización. Economía y Desarrollo, 3(1), 53-67.

Sen, A. (2003). La economía política de la focalización. Comercio Exterior, 53(6), 555562. http://revistacomercioexterior.com/rce/magazines/53/7/RCE.pdf

Sojo, A. (2007). La trayectoria del vínculo entre políticas selectivas contra la pobreza y políticas sectoriales. Revista de la CEPAL, 91, 111-131. 